\title{
Editors' Choice of Noteworthy Clinical Endoscopy Publications in the First Decade
}

\author{
Gwang Ha Kim ${ }^{1 *}$, Kwang An Kwon ${ }^{2}$, Do Hyun Park ${ }^{3}$ and Jimin Han ${ }^{4}$ \\ ${ }^{1}$ Department of Internal Medicine, Pusan National University School of Medicine and Biomedical Research Institute, Pusan National \\ University Hospital, Busan, ${ }^{2}$ Department of Gastroenterology, Gachon University Gil Hospital, Incheon, ${ }^{3}$ Division of Gastroenterology, \\ Department of Internal Medicine, Asan Medical Center, University of Ulsan College of Medicine, Seoul, ${ }^{4}$ Division of Gastroenterology, \\ Department of Internal Medicine, Daegu Catholic University School of Medicine, Daegu, Korea
}

This is a special review to celebrate the $10^{\text {th }}$ anniversary of Clinical Endoscopy. Each deputy editor has selected articles from one's subspecialty that are significant in terms of the number of downloads, citations, and clinical importance. The articles included original articles, review articles, systematic reviews, and meta-analyses. Clin Endosc 2021;54:633-640

\section{INTRODUCTION}

To commemorate the $10^{\text {th }}$ anniversary of Clinical Endosco$p y$, the deputy editors selected articles that were significant in terms of the number of downloads, citations, and clinical importance. The articles were selected from those reporting upper gastrointestinal disorders, lower gastrointestinal disorders, and pancreatobiliary disorders. They included original articles, review articles, systematic reviews, and meta-analyses.

\section{UPPER GASTROINTESTINAL DISORDERS}

\section{Endoscopic submucosal dissection}

Endoscopic submucosal dissection (ESD) has been widely accepted as the standard treatment for early gastric cancers
(EGCs) without the risk of regional lymph node metastasis and gastric dysplasia, in that it allows en bloc resection and has the advantages of less invasiveness and comparable outcomes with surgical resection. However, there are several important issues regarding ESD for gastric neoplasms, including the occurrence of adverse events such as bleeding and perforation, non-curative resection, and synchronous and metachronous gastric neoplasm during and after ESD. Three papers that have been recently published in Clinical Endoscopy pertain to these issues. ${ }^{1-3}$ Yorita et al. reported the results of a pilot study on the usefulness of dual red imaging (DRI) during ESD. ${ }^{1}$ Bleeding control during this procedure is important for safe and successful ESD, but it is somewhat difficult with conventional white light imaging alone. Therefore, the authors used DRI, a new image-enhanced endoscopy technique, for the recognition of blood vessels in the submucosal layer. The visibility of

\footnotetext{
Received: August 5, 2021 Revised: August 26, 2021

Accepted: August 30, 2021

Correspondence: Jimin Han

Division of Gastroenterology, Department of Internal Medicine, Daegu Catholic University School of Medicine, 33 Duryugongwon-ro 17-gil, Nam-gu, Daegu 42472, Korea Tel: +82-53+650-3442, Fax: +82-53-656-3281, E-mail: jmhan@cu.ac.kr

ORCID: https://orcid.org/0000-0001-8674-370X

Correspondence: Do Hyun Park

Digestive Diseases Research Center, Department of Internal Medicine, University of Ulsan College of Medicine, Asan Medical Center, Seoul 05505, Korea

Tel: +82-2-3010-3180/3190, Fax: +82-2-476-0824/485-5782, E-mail: dhpark@amc.seoul.kr

ORCID: https://orcid.org/0000-0002-0270-6969

* These authors contributed equally to this study

(c) This is an Open Access article distributed under the terms of the Creative Commons Attribution Non-Commercial License (http://creativecommons.org/licenses/by$\mathrm{nc} / 3.0$ ) which permits unrestricted non-commercial use, distribution, and reproduction in any medium, provided the original work is properly cited.
} 
bleeding points was improved in 55\% (11/20) of cases with the use of DRI compared with white light imaging. These results suggest that the proper use of an image-enhanced endoscopy technique is helpful for the detection of blood vessels during ESD, resulting in a clean and successful operation.

Lee et al. reported the risk factors and clinical outcomes of non-curative resection in patients with EGC. ${ }^{2}$ In this case, additional treatment such as redo-ESD, argon plasma coagulation, or gastrectomy with lymph node (LN) dissection is needed because of local recurrence and LN metastasis; however, this clinical approach can differ according to the patients' condition such as old age, concomitant disease, poor general health, or refusal to undergo additional treatment. In this retrospective multicenter study, the authors included 661 patients who underwent non-curative resection after ESD for EGC. Multivariate analysis showed that the risk factors affecting non-curative resection were old age, undifferentiated histology, tumor location in the upper body, large tumor size $(\geq 2 \mathrm{~cm})$, and presence of an ulcer. Cox proportional hazard regression analysis showed that a large tumor size $(\geq 2 \mathrm{~cm})$, submucosal invasion, positive horizontal margin, and lymphovascular invasion were risk factors for local recurrence. There was no statistically significant difference in the overall survival between the curative and non-curative resection groups (10year survival rates: $97.1 \%$ vs. $96.8 \%, p=0.788$ by log-rank test). Disease-specific survival was statistically lower in the non-curative resection group (10-year survival rates: $99.7 \%$ vs. $99.3 \%$, $p=0.038$ by log-rank test), but the difference was very small. These results suggest that proper selection of patients who can achieve curative resection after ESD of EGCs should be emphasized, and an individualized approach to patients with non-curative resection could be considered on the basis of the clinicopathological status of patients.

The occurrence of synchronous and/or metachronous gastric tumors is an important issue in the curative ESD of gastrointestinal neoplasms. Lee et al. reported the characteristics of synchronous and metachronous gastric tumor after ESD of early gastric neoplasm in 643 patients. ${ }^{3}$ Forty-four (6.8\%) synchronous and 100 (15.5\%) metachronous tumors were detected during the mean follow-up duration of 46 months (range, 12-148 months). The cumulative incidence rate steadily increased in proportion to the follow-up period (18.5\% at 3 years, $29.6 \%$ at 5 years, $38.7 \%$ at 7 years, and $47.5 \%$ at 9 years). More than $50 \%$ of multiple tumors developed at the same longitudinal location of the stomach, and had the same macroscopic and histological type as the primary lesions. These results suggest that since the frequency of synchronous and/ or metachronous gastric tumors after ESD of gastric neoplasm is high, strict long-term post-ESD surveillance endoscopy is mandatory for at least up to 10 years.

\section{Endoscopic-laparoscopic cooperative surgery}

Since ESD does not remove the muscular layer or serosa of the stomach, ESD often fails to achieve microscopically margin-negative (R0) resection for gastric subepithelial tumors (SETs) and results in high rates of perforation. ${ }^{4}$ Endoscopic full thickness resection (EFTR) and laparoscopic-endoscopic cooperative surgery overcome these limitations of ESD. Hajer et al. reported the experience of non-exposure endoscopic-laparoscopic cooperative surgery for gastric tumors in a recent issue of Clinical Endoscopy. ${ }^{5}$ Non-exposure endoscopic wall-inversion surgery (NEWS) and a combination of laparoscopic and endoscopic approaches to neoplasia with a non-exposure technique (CLEAN-NET) were performed for 12 gastric tumors, mainly SETs. R0 resection was achieved in all cases, without any major intraoperative adverse events. The authors suggested that NEWS is preferable for tumors in the subcardial and pyloric regions of the stomach, and CLEANNET is preferred in tumors with diameters $>4 \mathrm{~cm}$. Although many techniques have been developed for EFTR, it has not been widely adopted in routine clinical practice because of the technical challenges faced by endoscopists using currently available devices. Endoscopic-laparoscopic cooperative surgery could be an alternative option for EFTR to some degree.

\section{Endoscopic treatment of gastrointestinal perforation, fistula, and anastomotic leakage}

Gastrointestinal (GI) perforation, fistulas, and anastomotic leakages are potential indications for endoscopic treatment. However, the success rates of endoscopic treatment with commonly used devices, such as histoacryl glue, through-thescope clips, endoloops, and covered metal stents, are highly variable and unsatisfactory. To overcome this limitation, endoscopic vacuum therapy and closure using an over-thescope clip system have been recently applied for the treatment of GI perforation, fistulas, and anastomotic leakages.$^{6,7}$ In the recent issues of Clinical Endoscopy, there are two articles on the use of an over-the-scope clip system in the field of endoscopic treatment. ${ }^{8.9}$ Lee et al. reported the efficacy of an overthe-scope clip system for the treatment of GI fistula, leaks, and perforation. ${ }^{8}$ This prospective multicenter study has used an over-the-scope clip called OTSC (Ovesco Endoscopy, Tübingen, Germany) and included 19 patients. The median size of the defect was $10 \mathrm{~mm}$ (range, 5-30 mm), and the median procedure time was 16 minutes (range, 10-30 minutes). All cases were technically successful, and complete healing was achieved in 14 of 19 patients (74\%) using OTSC alone. When patients were classified into two groups (perforation and leakage vs. fistula), the rate of successful closure of perforation and leakage was significantly higher than that of fistulas ( $87 \%$ vs. $25 \%, p=0.025)$. No adverse events were associated with OTSC 
procedures. Goenka et al. reported the efficacy of a novel overthe-scope clip, Padlock clip (Aponos Medical Co., Kingston, $\mathrm{NH}, \mathrm{USA}){ }^{9}$ The researchers included four patients with GI bleeding and three patients for endoscopic full-thickness resection of GI tumors. The technical success rate was $100 \%$, without any adverse events. The results of the above two studies suggest that the over-the-scope clip system is an effective treatment modality in the field of endoscopic full-thickness resection of GI tumors, as well as endoscopic treatment of GI defects and bleeding. ${ }^{8.9}$

\section{LOWER GASTROINTESTINAL DISORDERS}

\section{Bowel preparation}

Bowel preparation is necessary before colonoscopy and involves administering and taking medications to cleanse the colon. Colonoscopy is optimally performed only when the bowel is clean. Over the decades, various efforts have been made to improve bowel preparation, and one of them includes educating patients via smartphones. Moreover, a variety of contents can be developed using smartphones. Jeon et al. evaluated the effect of educational video clips on bowel preparation before colonoscopy. ${ }^{10} \mathrm{~A}$ total of 140 patients in the smartphone mobile messenger (SMM) group and 141 patients in the control group underwent colonoscopy. The total Ottawa score of the SMM group was significantly lower than that of the control group $(5.47 \pm 1.74$ vs. $5.97 \pm 1.78, p=0.018)$. These results were particularly prominent in young patients. The total Ottawa score of patients in the SMM group aged $<40$ years was significantly lower than that of patients in the control group aged $<40$ years $(5.10 \pm 1.55$ vs. $6.22 \pm 2.33, p=0.034)$.

\section{Diminutive colon polyps}

Ninety percent of polyps found during colonoscopy measure less than $1 \mathrm{~cm}$ in size, and hyperplastic polyps measuring less than $5 \mathrm{~mm}$ account for a significant portion of polyps found. The duration of follow-up after resection of small polyps is determined by their number, size, and pathology. Recently, with the increase in the number of colonoscopies, the number of polyps found has increased, and optical diagnostic technology is being developed. This interesting review by Kandel et al. focused on the cost-effectiveness of whether diagnosing using optical devices can replace pathology reports. ${ }^{11}$ The authors reviewed the "resect and discard" strategies for small colon polyps. The American Society for Gastrointestinal Endoscopy Preservation and Incorporation of Valuable Endoscopic Innovations support the strategies for optical diagnosis of small non-neoplastic polyps, as long as two criteria are met.
For hyperplastic polyps measuring $<5 \mathrm{~mm}$ in the rectosigmoid colon, the negative predictive value should be at least $90 \%$. For diminutive low-grade adenomatous polyps, a "resect and discard" strategy should be sufficiently accurate such that the post-polypectomy surveillance recommendations based on the optical diagnosis agree with those based on the histological diagnosis at least $90 \%$ of the time. Although the "resect and discard" strategy and the "diagnose and leave behind" approach have major benefits regarding both safety and cost, they are yet to be used widely in practice.

\section{Colorectal endoscopic submucosal dissection}

With the development of colorectal ESD, large mucosal colon tumors and superficial submucosal tumors can now be removed without surgery. Because LN metastasis is affected by submucosal tumor invasion, it is important to determine the depth of tumor invasion before endoscopic removal of colorectal cancer. Lee et al. reviewed various methods for estimating the invasion depth of malignant colorectal polyps prior to colorectal ESD. ${ }^{12}$ Among gross findings, narrow-band imaging (NBI), magnifying chromoendoscopy, endoscopic ultrasonography, endocytoscopy, artificial intelligence, and the Japan NBI Expert Team (JNET) classification of NBI magnifying endoscopy findings are useful methods for histological prediction and invasion depth estimation. However, magnifying chromoendoscopy is still necessary for JNET type 2B lesions to achieve satisfactory diagnostic accuracy.

\section{Colon capsule endoscopy}

Colonoscopy is still the standard method for diagnosing and treating colorectal diseases. Colon capsule endoscopy (CCE) can be an alternative method when colonoscopy is difficult or incomplete for various reasons. Advances in CCE have been made with the development of technology. Hong et al. reviewed the advances and indications for CCE. ${ }^{13}$ The authors presented the reasons for not using CCE widely at present and the challenges to be solved in the future. CCE is a convenient, noninvasive method that enables physicians to examine the entire colon without causing significant discomfort to the patient. Although CCE can be performed painlessly without bowel air insufflation, vigorous bowel preparation and other technical limitations exist. Therefore, CCE has not yet replaced conventional colonoscopy.

\section{Colorectal cancer screening}

Colon cancer originates mostly from colon polyps, and removing colon polyps can prevent the progression of colon cancer. The incidence of colon cancer is also reportedly low in the group that has been screened for colon cancer. ${ }^{14}$ The standards for colorectal cancer screening vary by age and country. 
Bevan et al. reviewed the currently available screening methods for colorectal cancer (CRC), most of which are diagnosed after 60 years of age, and most screening programs are applicable to individuals aged $50-75$ years. ${ }^{15}$ Screening may reduce disease-specific mortality by detecting CRC at an early stage and the incidence of CRC by detecting premalignant polyps, which may subsequently be removed. In randomized controlled trials (RCTs), guaiac fecal occult blood testing (gFOBt) was found to reduce CRC mortality by $13 \%-33 \%$. No RCTs data is available comparing fecal immunochemical testing to no screening, but it is superior to gFOBt. Trials focusing on flexible sigmoidoscopy-based screening demonstrated an 18\% reduction in the incidence of CRC and a $28 \%$ reduction in CRC mortality.

\section{Fecal microbiota transplantation}

Intestinal microbial imbalance is presumably related to inflammatory bowel disease, autoimmune diseases, allergies, and metabolic diseases such as obesity. Therefore, efforts to treat the disease through fecal transplantation are being made, and a practical consideration of the selection of the target patient, stool donor, procedure, and patient management will be helpful. Kim et al. reviewed the recent advancements in fecal microbiota transplantation (FMT). ${ }^{16}$ FMT has been successfully used to treat recurrent Clostridium difficile infection (rCDI). The short-term success of FMT in treating rCDI has prompted investigations into its application to other gastrointestinal disorders and extra-intestinal diseases with presumed gut dysbiosis. Although FMT showed promising results in the treatment of these conditions, there are still several challenges for its application, including characterization of a healthy microbiome, ensuring recipient safety with respect to longterm outcomes, adequate monitoring of the recipient for fecal material, achieving high-quality control, and maintaining reasonable costs.

\section{Artificial intelligence and robotics in endoscopy}

Artificial intelligence is making significant strides by learning the characteristics and concepts of objects from machine learning and deep learning methods. Artificial intelligence is here now, so it is important to learn about its basic mechanisms and latest application technologies, especially in the medical field. Robotic colonoscopy with efficient locomotion has been developed to reduce complications such as bleeding and perforation compared to conventional colonoscopy, reduce pain in patients, and lessen the physical fatigue of endoscopists.

Small-bowel capsule endoscopy is widely used for investigating obscure gastrointestinal bleeding from a source that cannot be identified using upper or lower endoscopy, and the methods for reading data have greatly improved. However, endoscopic experts still need to spend a lot of time analyzing the captured images. Therefore, the introduction of advanced artificial intelligence for analyzing small bowel capsule endoscopic images is particularly interesting. Hwang et al. reviewed the application of artificial intelligence in capsule endoscopy. ${ }^{17}$ Capsule endoscopy requires a clinical specialist to spend additional time to review the operation and examine the lesions. Various approaches for computer-aided diagnosis have been reported based on artificial intelligence to reduce the review time and increase the accuracy of medical examinations. Recently, deep learning-based approaches have been applied in possible areas, showing greatly improved performance, especially for image-based recognition and classification.

Abadir et al. reviewed the applications of artificial intelligence in gastrointestinal endoscopy ${ }^{18}$ The authors described the development of the basic mechanisms of machine learning and convolutional neural networks in artificial intelligence. This in-depth review focused on the applications of artificial intelligence in colonoscopy, inflammatory bowel disease, early gastric cancer detection, esophageal neoplasia detection, limitations of artificial intelligence use, and problems to be solved in the future. In the field of gastroenterology, artificial intelligence has been studied as a tool to assist in risk stratification, diagnosis, and pathological identification. Specifically, in the field of endoscopy, artificial intelligence has attracted great interest as a technology with the potential to revolutionize the practice of modern gastroenterology. From cancer screening to automated report generation, artificial intelligence has touched all aspects of modern endoscopy.

Wong et al. reviewed the application of robotics in advanced colonoscopy. ${ }^{19}$ They focused on the role of robotics in colonoscopy to reduce endoscopists' fatigue. The researchers also described different robot systems developed around the world and discussed their future in detail. Among the recent developments, highly dexterous robotic master and slave systems, computer-assisted or robotically-enhanced conventional endoscopes, and autonomously-driven locomotion devices can effortlessly traverse the colon. Developments in endoscopic instrumentation have overcome technical barriers and have opened new avenues for further advancements in therapeutic interventions.

\section{PANCREATOBILIARY DISORDERS}

\section{EUS-guided tissue acquisition for pancreatic masses}

Endoscopic ultrasound (EUS)-guided tissue acquisition has become the standard of care for the diagnosis of peri- and pancreatic masses. ${ }^{20}$ In 2020, two remarkable studies that focused 
on EUS-guided tissue acquisition on pancreatic masses were published. ${ }^{21,22}$ Cui et al. studied the role of EUS-guided Ki67 in the management of non-functioning pancreatic neuroendocrine tumors (NF-PNETs). ${ }^{21}$ As usual, small PNETs (less than $2 \mathrm{~cm}$ ) were managed by regular follow-up rather than surgical resection owing to the favorable prognosis of small PNETs and invasiveness of surgical resection. ${ }^{23}$ This study investigated the concordance rate of Ki67 grading between EUS-guided fine-needle aspiration (EUS-FNA) and surgical pathology specimens in NF-PNETs and whether certain NF-PNETs characteristics are associated with disease recurrence and disease-related death. There was $73 \%$ concordance between Ki67 obtained from EUS-FNA cytology and surgical pathology specimens; concordance was highest for low- and highgrade NF-PNETs. They concluded that NF-PNETs with highgrade Ki67 on EUS-FNA had poor prognosis despite surgical resection. NF-PNETs with intermediate-grade Ki67 on EUSFNA should be considered for surgical resection. NF-PNETs with low-grade Ki67 on EUS-FNA can be monitored without surgical intervention, up to a tumor size of $20 \mathrm{~mm}$. However, several reports on the considerable discordance rate between EUS-guided FNA and surgical pathology on Ki67 grading of PNETs have been published. Therefore, the results from a mono-centric retrospective study should be interpreted with caution. Larger prospective multicenter studies are required to confirm this important issue.

de Moura et al. evaluated the comparative efficacy and safety of FNA versus fine-needle biopsy (FNB) for LN sampling. ${ }^{22}$ This was a multicenter retrospective study of prospectively collected data to evaluate the outcomes of EUS-FNA and EUSFNB for LN sampling. This study showed similar sensitivity and accuracy between FNA and FNB (sensitivity: 67.21\% vs. $75.00 \%$, respectively, $p=0.216$ and accuracy: $78.80 \%$ vs. $83.17 \%$, respectively, $p=0.423$ ). The specificity of FNB was better than that of FNA $(100.00 \%$ vs. $93.62 \%, p=0.01)$. The number of passes required for diagnosis was not different. FNB of abdominal and peri-hepatic LN showed higher sensitivity $(81.08 \%$ vs. $64.71 \%, p=0.031$ and $80.95 \%$ vs. $58.33 \%$, $p=0.023)$ and accuracy $(88.14 \%$ vs. $75.29 \%, p=0.053$ and $88.89 \%$ vs. $70.49 \%, p=0.038$ ) when compared to those of FNA, each respectively. Rapid on-site examination (ROSE) was a significant predictor of accuracy (odds ratio, 5.16; 95\% confidence interval, 1.15-23.08; $p=0.032$ ). No adverse events were reported in either cohort. EUS-FNA+ ROSE was similar to EUS-FNB alone, showing a better diagnosis for EUS-FNB than traditional FNA. While ROSE remained a significant predictor for accuracy, due to its poor availability in most centers, its use may be limited to cases with previous inconclusive diagnoses. A recent multicenter randomized controlled trial comparing EUS-FNA with ROSE and EUS-FNB for pancreat- ic masses showed similar efficacies in both methods. ${ }^{20}$ Therefore, EUS-FNB can be performed without ROSE.

\section{EUS-guided tissue acquisition for biliary strictures and liver}

For indeterminate biliary stricture, the utility of EUS-guided tissue acquisition may have an important role because of the low sensitivity of endoscopic retrograde cholangiopancreatography (ERCP)-guided tissue sampling, especially brush cytol$\mathrm{ogy}^{24}$ de Moura et al. performed a meta-analysis of same-session procedures with EUS-guided fine-needle aspiration and ERCP-based tissue sampling for suspected malignant biliary stricture. ${ }^{25}$ In this meta-analysis, the sensitivity, specificity, positive likelihood ratio, negative likelihood ratio, and accuracy of the association between the two methods were $86 \%, 98 \%$, $12.50 \%, 0.17 \%$, and $96.5 \%$, respectively. For the individual analysis, the sensitivity, specificity, and accuracy of EUS-FNA were $76 \%, 100 \%$, and $94.5 \%$, respectively. For ERCP-based tissue sampling, the sensitivity, specificity, and accuracy were $58 \%, 98 \%$, and $78.1 \%$, respectively. EUS-FNA was superior to ERCP-based tissue sampling for pancreatic lesions. However, for biliary lesions, both methods had similar sensitivities. Same-session EUS-FNA and ERCP-based tissue sampling may be superior to either method alone in the diagnosis of suspected malignant biliary strictures. ${ }^{25}$ However, there is the risk of tumor seeding after EUS-FNA for proximal biliary stricture rather than distal biliary stricture. ${ }^{24,26,27}$ Therefore, further larger prospective studies may be required for this concern.

McCarty et al. performed systematic review and meta-analysis for EUS-guided liver biopsy (EUSLB), percutaneous liver biopsy (PCLB), and transjugular liver biopsy (TJLB) ${ }^{28}$ Biopsy cumulative adequacy rates for EUSLB, PCLB, and TJLB were $93.51 \%, 98.27 \%$, and $97.61 \%$, respectively. A comparison of EUSLB and PCLB revealed no difference between specimens regarding both complete portal triad (CPT) $(p=0.079)$ and length of longest piece (LLP) $(p=0.085)$; however, a longer total specimen length (TSL) $(p<0.001)$ was observed in EUSLB. Compared to TJLB, EUSLB showed no difference in LLP $(p=0.351)$, fewer CPT $(p=0.042)$, and longer TSL $(p=0.005)$. EUSLB appears to be a safe, minimally invasive procedure comparable to PCLB and TJLB regarding biopsy specimens obtained and the rate of adverse events associated with each method. Recently, a randomized trial comparing EUSLB and PCLB showed that PCLB yielded significantly more optimal specimens, defined as a specimen length of $25 \mathrm{~mm}$ or greater and the presence of at least $11 \mathrm{CPTs}(57.9 \%$ vs. $23.8 \%$, $p=0.028) .{ }^{29}$ However, the standardized EUSLB has not been studied so far compared to PCLB. Further larger multicenter studies on this issue are required. 


\section{Lumen-apposing metal stent for peripancreatic fluid collections}

Recently, lumen-apposing metal stents (LAMSs) have been widely used for EUS-guided drainage of peripancreatic fluid collections (PFCs) and infected walled-off necrosis. ${ }^{30,31}$ Most studies were conducted in an academic hospital setting. Therefore, the outcomes of LAMSs in community hospital settings are lacking. In the community hospital-based retrospective study by Garg et al., 27 patients, with a mean age of $54.1 \pm 6.5$ years, were included, $44 \%$ of whom were men. The mean size of the PFCs was $9.7 \pm 5.0 \mathrm{~cm}$ (range, $3-21$ ). ${ }^{32}$ The most common etiology of pancreatitis was alcohol (44\%) followed by idiopathic causes (30\%) and presence of gallstones (22\%). The diagnosis was pseudocyst in $44.4 \%$ (12/27) and walledoff necrosis in $55.6 \%(15 / 27)$ of patients. The technical success rate was $100 \%$ without any complications. Clinical success was achieved in 22 of 27 patients (81.5\%) who underwent stent removal. This is the first study to report that endoscopic therapy of PFCs using LAMSs is safe and effective even in a community hospital setting with limited resources and support compared to large academic centers.

Recently, various types of LAMSs have been introduced. Shin et al. compared the clinical outcomes between a plastic stent and novel LAMSs for EUS-guided drainage of PFCs. ${ }^{33}$ Twenty-seven patients (median age, 56 years) with PFCs underwent EUS-guided transmural drainage, with 17 for plastic stent (PS) placement and 10 for LAMSs placement. There was no significant difference in the technical success rate between the two groups ( $94.1 \%$ vs. $100 \%, p=1.0$ ). Procedure time was shorter in the LAMSs group than in the PS group $(10.6 \pm 2.5$ $\min$ vs. $21.4 \pm 9.5 \mathrm{~min}, p=0.002)$. Among subjects with clinical success, recurrence of PFC after stent removal occurred in 5 of 12 patients with PS and 4 of 10 with LAMSs, without statistical difference $(41.7 \%$ vs. $40.0 \%, p=1.0) .^{33}$ Although this study showed similar clinical outcomes for LAMSs and PS, further prospective trials are required to validate the superiority of LAMSs because of the retrospective design and small patient population in this single study.

\section{Novel transpapillary methods for perihilar biliary obstruction and acute cholecystitis}

Endoscopic palliation of perihilar biliary obstruction seems challenging because of frequent clogging of plastic stents and tumor ingrowth in uncovered metal stents. ${ }^{34,35}$ Kanno et al. evaluated the outcomes of inside plastic stents (iPSs) versus those of metal stents (MSs) for treating unresectable perihilar malignant obstructions. ${ }^{36}$ For all patients who underwent endoscopic suprapapillary placement of iPSs or MSs as the first permanent biliary drainage for unresectable malignant perihilar obstructions, there were no differences in clinical effectiveness (95\% for the iPSs group vs. $92 \%$ for the MSs group, $p=1.00$ ). Procedure-related adverse events, including pancreatitis, acute cholangitis, acute cholecystitis, and death, were observed in $8 \%$ of the MSs group, although no patient in the iPSs group experienced such adverse events. The median time to recurrent biliary obstruction (RBO) was 561 days (95\% confidence interval, 0-1,186 days) for iPSs and 209 days (127291 days) for MSs, showing a significant difference $(p=0.008){ }^{36}$ Time to RBO after iPSs placement was significantly longer than that after MSs placement. IPSs, which are removable, unlike MSs, are an acceptable option. Given these promising results, further randomized trials comparing iPSs and MSs for the palliation of unresectable perihilar malignant biliary obstruction are needed.

Endoscopic transpapillary gallbladder drainage (ETGBD) is useful for the treatment of acute cholecystitis in patients who are unsuitable for cholecystectomy; however, this technique is difficult to perform. ${ }^{37}$ When intraductal ultrasonography (IDUS) is combined with ETGBD, the orifice of the cystic duct in the common bile duct may be more easily detected in the cannulation procedure. Sagami et al. studied the efficacy of ETGBD with IDUS compared with that of ETGBD alone. ${ }^{38}$ One-hundred consecutive patients with acute cholecystitis requiring ETGBD were retrospectively recruited. The first 50 consecutive patients were treated using ETGBD without IDUS, and the next 50 were treated using ETGBD with IDUS. The technical success rate of ETGBD with IDUS was significantly higher than that without IDUS $(92.0 \%$ vs. $76.0 \%, p=0.044)$. There was no significant difference in procedure length between the two groups $(74.0 \mathrm{~min}$ vs. $66.7 \mathrm{~min}$, $p=0.310$ ). The complication rate of ETGBD with IDUS was significantly higher than that of ETGBD without IDUS (6.0\% vs. $0 \%, p<0.001$ ); however, only one case showed an IDUS technique-related complication (pancreatitis). ${ }^{38}$ The assistance of IDUS may be useful in ETGBD. Single-operator peroral cholangioscopy may be another option for patients with difficult cystic access in ETGBD. ${ }^{39}$ Comparative studies on IDUS and single operator cholangioscopy for access to the cystic duct in ETGBD would be of interest.

\section{CONCLUSIONS}

In this special review celebrating the $10^{\text {th }}$ anniversary of Clinical Endoscopy, the deputy editors assembled notable articles to demonstrate the past and present. Since each article published in Clinical Endoscopy is remarkable, the process was not easy. The success of Clinical Endoscopy stems from the researchers who submit their valuable manuscripts, the endeavors of the editorial board members, and the devoted readers. 
The Editorial Board of Clinical Endoscopy sincerely appreciates everyone's contribution and commitment and will continue to deliver informative articles for doctors and other healthcare personnel involved in endoscopic procedures.

\section{Conflict of Interest}

Gwang Ha Kim, Kwang An Kwon, and Do Hyun Park are currently serving as deputy editors in Clinical Endoscopy; however, they were not involved in the peer reviewer selection, evaluation, or decision process of this article. Jimin Han is currently serving as an associate editor in Clinical Endoscopy; however, Jimin Han was not involved in the peer reviewer selection, evaluation, or decision process of this article. The authors have no potential conflicts of interest.

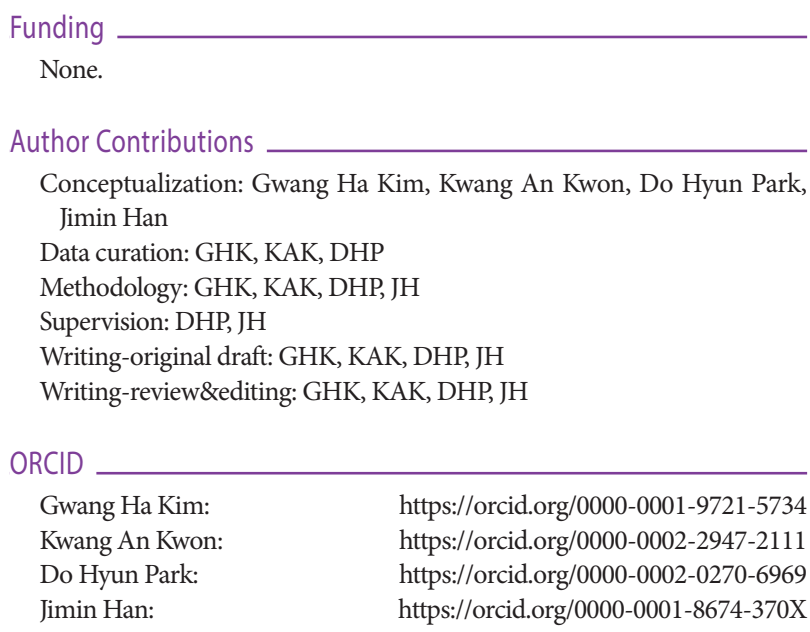

\section{REFERENCES}

1. Yorita N, Oka S, Tanaka S, et al. Clinical usefulness of dual red imaging in gastric endoscopic submucosal dissection: a pilot study. Clin Endosc 2020;53:54-59.

2. Lee SH, Kim MC, Jeon SW, Lee KN, Park JJ, Hong SJ. Risk factors and clinical outcomes of non-curative resection in patients with early gastric cancer treated with endoscopic submucosal dissection: a retrospective multicenter study in Korea. Clin Endosc 2020;53:196-205.

3. Lee HJ, Lee YJ, Lee JY, et al. Characteristics of synchronous and metachronous multiple gastric tumors after endoscopic submucosal dissection of early gastric neoplasm. Clin Endosc 2018;51:266-273.

4. Kaan HL, Ho KY. Endoscopic full thickness resection for gastrointestinal tumors - challenges and solutions. Clin Endosc 2020;53:541-549.

5. Hajer J, Havlůj L, Whitley A, Gürlich R. Non-exposure endoscopic-laparoscopic cooperative surgery for stomach tumors: first experience from the Czech Republic. Clin Endosc 2018;51:167-173.

6. Jung DH, Yun H-R, Lee SJ, Kim NW, Huh CW. Endoscopic vacuum therapy in patients with transmural defects of the upper gastrointestinal tract: a systematic review with meta-analysis. J Clin Med 2021;10:23462360.

7. Haito-Chavez Y, Law JK, Kratt T, et al. International multicenter experience with an over-the-scope clipping device for endoscopic management of GI defects (with video). Gastrointest Endosc 2014;80:610-622.

8. Lee HL, Cho JY, Cho JH, et al. Efficacy of the over-the-scope clip system for treatment of gastrointestinal fistulas, leaks, and perforations: a korean multi-center study. Clin Endosc 2018;51:61-65.
9. Goenka MK, Rodge GA, Tiwary IK. Endoscopic management with a novel over-the-scope padlock clip system. Clin Endosc 2019;52:574-580.

10. Jeon SC, Kim JH, Kim SJ, et al. Effect of sending educational video clips via smartphone mobile messenger on bowel preparation before colonoscopy. Clin Endosc 2019;52:53-58.

11. Kandel P, Wallace MB. Should we resect and discard low risk diminutive colon polyps. Clin Endosc 2019;52:239-246.

12. Lee BI, Matsuda T. Estimation of invasion depth: the first key to successful colorectal ESD. Clin Endosc 2019;52:100-106.

13. Hong SN, Kang SH, Jang HJ, Wallace MB. Recent advance in colon capsule endoscopy: what's new? Clin Endosc 2018;51:334-343.

14. Thiis-Evensen E, Hoff GS, Sauar J, Langmark F, Majak BM, Vatn MH. Population-based surveillance by colonoscopy: effect on the incidence of colorectal cancer. Telemark Polyp Study I. Scand J Gastroenterol 1999;34:414-420.

15. Bevan R, Rutter MD. Colorectal cancer screening-who, how, and when? Clin Endosc 2018;51:37-49.

16. Kim KO, Gluck M. Fecal microbiota transplantation: an update on clinical practice. Clin Endosc 2019;52:137-143.

17. Hwang Y, Park J, Lim YJ, Chun HJ. Application of artificial intelligence in capsule endoscopy: where are we now? Clin Endosc 2018;51:547-551.

18. Abadir AP, Ali MF, Karnes W, Samarasena JB. Artificial intelligence in gastrointestinal endoscopy. Clin Endosc 2020;53:132-141.

19. Wong JYY, Ho KY. Robotics for advanced therapeutic colonoscopy. Clin Endosc 2018;51:552-557.

20. Crinò SF, Di Mitri R, Nguyen NQ, et al. Endoscopic ultrasound-guided fine-needle biopsy with or without rapid on-site evaluation for diagnosis of solid pancreatic lesions: a randomized controlled non-inferiority trial. Gastroenterology 2021;161:899-909.e5.

21. Cui Y, Khanna LG, Saqi A, et al. The role of endoscopic ultrasound-guided Ki67 in the management of non-functioning pancreatic neuroendocrine tumors. Clin Endosc 2020;53:213-220.

22. de Moura DTH, McCarty TR, Jirapinyo P, et al. Endoscopic ultrasound fine-needle aspiration versus fine-needle biopsy for lymph node diagnosis: a large multicenter comparative analysis. Clin Endosc 2020;53:600610.

23. Choi JH, Park DH, Kim MH, et al. Outcomes after endoscopic ultrasound-guided ethanol-lipiodol ablation of small pancreatic neuroendocrine tumors. Dig Endosc 2018;30:652-658.

24. Jo JH, Cho CM, Jun JH, et al. Same-session endoscopic ultrasound-guided fine-needle aspiration and endoscopic retrograde cholangiopancreatography-based tissue sampling in suspected malignant biliary obstruction: a multicenter experience. J Gastroenterol Hepatol 2019;34:799-805.

25. de Moura DTH, Ryou M, de Moura EGH, Ribeiro IB, Bernardo WM, Thompson CC. Endoscopic ultrasound-guided fine-needle aspiration and endoscopic retrograde cholangiopancreatography-based tissue sampling in suspected malignant biliary strictures: a meta-analysis of same-session procedures. Clin Endosc 2020;53:417-428.

26. Parsa N, Khashab MA. The Role of peroral cholangioscopy in evaluating indeterminate biliary strictures. Clin Endosc 2019;52:556-564.

27. So H, Park DH. Digital single-operator cholangioscopy for indeterminate biliary stricture: enthusiasm or still evolving for unmet need? Gastrointest Endosc 2020;91:394-395.

28. McCarty TR, Bazarbashi AN, Njei B, Ryou M, Aslanian HR, Muniraj T. Endoscopic ultrasound-guided, percutaneous, and transjugular liver biopsy: a comparative systematic review and meta-analysis. Clin Endosc 2020;53:583-593.

29. Bang JY, Ward TJ, Guirguis S, et al. Radiology-guided percutaneous approach is superior to EUS for performing liver biopsies. Gut 2021;gutjnl-2021-324495,

30. Guzmán-Calderón E, Chacaltana A, Díaz R, Li B, Martinez-Moreno B, Aparicio JR. Head-to-head comparison between endoscopic ultrasound guided lumen apposing metal stent and plastic stents for the treatment of pancreatic fluid collections: a systematic review and meta-analysis. J Hepatobiliary Pancreat Sci 2021 Jun 9 [Epub]. https://doi.org/10.1002/ jhbp.1008. 
31. Siddiqui AA, Adler DG, Nieto J, et al. EUS-guided drainage of peripancreatic fluid collections and necrosis by using a novel lumen-apposing stent: a large retrospective, multicenter U.S. experience (with videos). Gastrointest Endosc 2016;83:699-707.

32. Garg R, Chaar A, Szpunar S, Mohan BP, Barawi M. Efficacy and safety of lumen-apposing stents for management of pancreatic fluid collections in a community hospital setting. Clin Endosc 2020;53:480-486.

33. Shin HC, Cho CM, Jung MK, Yeo SJ. Comparison of clinical outcomes between plastic stent and novel lumen-apposing metal stent for endoscopic ultrasound-guided drainage of peripancreatic fluid collections. Clin Endosc 2019;52:353-359.

34. So $\mathrm{H}$, Oh D, Takenaka M, et al. Initial experience of endoscopic ultrasound-guided antegrade covered stent placement with long duodenal extension for malignant distal biliary obstruction (with video). J Hepatobiliary Pancreat Sci 2021 Jun 12 [Epub]. https://doi.org/10.1002/ jhbp.1011.
35. Lee TH. Technical tips and issues of biliary stenting, focusing on malignant hilar obstruction. Clin Endosc 2013;46:260-266.

36. Kanno Y, Koshita S, Ogawa T, et al. Inside plastic stents versus metal stents for treating unresectable malignant perihilar biliary obstructions: a retrospective comparative study. Clin Endosc 2020;53:735-742.

37. Lee TH, Park DH, Lee SS, et al. Outcomes of endoscopic transpapillary gallbladder stenting for symptomatic gallbladder diseases: a multicenter prospective follow-up study. Endoscopy 2011;43:702-708.

38. Sagami R, Hayasaka K, Ujihara T, et al. A new technique of endoscopic transpapillary gallbladder drainage combined with intraductal ultrasonography for the treatment of acute cholecystitis. Clin Endosc 2020;53:221-229.

39. Shin JU, Lee JK, Kim KM, Lee KH, Lee KT. Endoscopic naso-gallbladder drainage by using cholangioscopy for acute cholecystitis combined with cholangitis or choledocholithiasis (with video). Gastrointest Endosc 2012;76:1052-1055 